\title{
Imaging of coronary flow capacity: is there a role for dynamic CT perfusion imaging?
}

\author{
Alexia Rossi $i^{1,2} \cdot$ Giuseppe Ferrante ${ }^{1,3}$
}

Received: 29 April 2019 / Accepted: 13 May 2019 / Published online: 31 May 2019

(C) Springer-Verlag GmbH Germany, part of Springer Nature 2019

The assessment of myocardial ischaemia in patients with coronary artery disease (CAD) being considered for myocardial revascularization procedures is of paramount importance. The detection of a large area of myocardial ischaemia by functional imaging is associated with impaired prognosis of patients and identifies patients who should undergo revascularization [1]. Non-invasive functional evaluation of myocardial ischaemia should be the first approach and can be achieved with a variety of techniques.

Nevertheless, invasive coronary pressure-derived fractional flow reserve (FFR) is the current standard of care for the functional assessment of angiographically intermediate-grade stenosis (typically around 40-90\% stenosis) without evidence of ischaemia in non-invasive testing, or in those with multivessel disease.

In the FAME 2 trial, percutaneous coronary intervention (PCI), as compared to medical therapy, was associated with lower incidence of the primary composite endpoint of death, myocardial infarction, and urgent revascularization in patients with stable CAD and at least one stenosis with FFR $\leq 0.80$ [2]. Recently, two large-scale randomized trials showed broadly comparable results between FFR-guided and the resting index instantaneous wave-free ratio

This article is part of the Topical Collection as an Editorial.

Alexia Rossi

alexia.rossi@hunimed.eu

1 Department of Biomedical Sciences, Humanitas Clinical and Research Hospital, Humanitas University, Pieve Emanuele, Milan, Italy

2 Department of Diagnostic Imaging, Humanitas Clinical and Research Hospital - IRCCS, Rozzano, Milan, Italy

3 Department of Cardiovascular Medicine, Humanitas Clinical and Research Hospital - IRCCS, Rozzano, Milan, Italy
(iwFR)-guided revascularization strategies in patients with intermediate-grade stenosis [3, 4].

Myocardial blood flow and coronary flow reserve (CFR, i.e. the ratio between hyperemic to rest flow) are the critical determinants of myocardial ischaemia. Invasive pressurederived FFR, originally validated by comparison to stress positron emission tomography (PET), is the derivative approximation of the relative regional distribution of stress perfusion expressed as a fraction of maximum stress perfusion in $\mathrm{ml} /$ $\mathrm{min} / \mathrm{g}[5]$.

Coronary flow capacity (CFC), originally developed using PET, integrates the simultaneous regional size severity of resting myocardial blood flow (MBF), hyperemic $\mathrm{MBF}$, and CFR [6]. CFC maps provide specific patterns that are more accurate than CFR for distinguishing the effects of focal CAD, diffuse non-obstructive CAD and microvascular dysfunction by accounting for perfusion heterogeneity [7]. Severely reduced CFC, defined as the coexistence of stress $\mathrm{MBF} \leq 0.91 \mathrm{ml} / \mathrm{min} / \mathrm{g}$ and $\mathrm{CFR} \leq 1.74$, has been shown to predict a significant reduction in death or myocardial infarction after revascularization compared with medical treatment or less severe perfusion abnormalities [7].

In the current issue of the Journal, Bober et al. assessed the effects of coronary revascularization by percutaneous coronary intervention on stress MBF by PET [8]. Fifty patients, who underwent clinically indicated dipyridamole myocardial ${ }^{82} \mathrm{Rb}$ PET, were enrolled in the study after coronary revascularization. A follow-up dipyridamole myocardial ${ }^{82} \mathrm{Rb}$ PET was performed within 90 days from coronary revascularization. For image analysis the left ventricle was divided into four quadrants corresponding to the distribution of the coronary arteries: anterior, septal, lateral and inferior [9]. Each quadrant was visually inspected for the presence of baseline significant relative perfusion abnormality (PA) defined as $\geq 10 \%$ change in size and/or severity from the resting scan. In addition, rest and stress MBF, CFR and $\mathrm{CFC}$ were calculated for each quadrant. Four different 
patterns were reported according to the presence of PA and severe reduction in CFC: (1) normal (PA-/CFC-), (2) severely abnormal $(\mathrm{PA}+/ \mathrm{CFC}+)$, (3) flow capacity abnormal (PA-/CFC+), and (4) perfusion abnormal (PA+/CFC-).

The main findings of this study can be summarized as follows: (1) severely reduced CFC at baseline was found to be the only independent predictor of significant improvement of stress MBF after including baseline PA, stress $\mathrm{MBF}$ and CFR in the multivariable regression model. In particular, stress MBF improved by 59\% when relative PA and reduced CFC were present at baseline and by $40 \%$ when baseline CFC only was reduced in the absence of a significant relative PA; (2) stress MBF did not change significantly after revascularization if baseline relative PA only was reduced and CFC was within normal limits, therefore underscoring the poor accuracy of relative PA as stand-alone perfusion metric for the identification of patients who may benefit from revascularization; (3) in a small subgroup of nine patients undergoing FFR-guided revascularization using an FFR threshold of $<0.80$, there was high discordance between FFR and CFC, as only one of the six quadrants with normal relative perfusion images and preserved CFC demonstrated improvement in stress MBF.

The authors should be congratulated on performing this elegant study which is the first to investigate the value of PET-derived CFC in predicting MBF response to coronary revascularization. Nevertheless, the decision to proceed to PCI was mainly based on angiographic evaluation of the severity of a coronary stenosis, and the study lacks clinical outcome data. Yet, the study findings support future prospective randomized clinical trials comparing PET-derived CFC-guided revascularization vs FFR or iw-FR guided revascularization in terms of clinical outcomes among patients with stable CAD and angiographically intermediategrade stenosis.

Although CFC was first validated using PET technology [6] and invasive coronary flow measurements by Doppler wire [10], the concept of CFC can be applied to all imaging modalities which allow the absolute quantification of myocardial perfusion. In this perspective, dynamic myocardial computed tomography perfusion (CTP) imaging is a noninvasive imaging modality which provides absolute quantification of MBF. For quantitative CTP imaging, serial sampling is performed to evaluate the iodine distribution into the myocardium and into a reference artery at different time points enabling the construction of time-attenuation curves (TACs). Applying specific mathematical models to the TACs, perfusion parameters, such as MBF, can be calculated. The feasibility of dynamic CTP was initially demonstrated in few animal studies [11-13]. Although in experimentally controlled settings CT-derived MBF showed an excellent correlation with microspheres [11], invasive fractional flow reserve [12] and coronary blood flow [12], first pass extraction of iodinated contrast material is generally low, especially for high flow rates, mainly due to the limited temporal sampling, with consequent underestimation of MBF [14, 15]. Despite this limitation, dynamic CTP succeeded in demonstrating the wellknown transmural blood flow gradient through the myocardium, showing lower endocardial values of CT-derived MBF in the ischaemic territories compared to the corresponding epicardial measurements [16]. In addition, Ho et al. showed that CT-derived stress MBF and CFR were higher in a low-risk population compared to patients with documented coronary artery disease [17] with a 1.5 difference between stress and rest MBF in normal myocardium [18]. Promising results on the ability of dynamic CTP in detecting myocardial ischaemia have been also shown in selected groups of symptomatic patients with suspected or known CAD with a pooled sensitivity and specificity on a vessel-based analysis of $85 \%$ and $81 \%$, respectively [19].

Quantitative imaging has introduced new diagnostic opportunities, first of which is evaluation of the status of the microcirculation. Specifically, a significant reduction of stress MBF associated with the absence of obstructive coronary artery disease is likely indicative of microvascular dysfunction. As such, a CT-based approach can offer both the anatomical information regarding the coronary tree and the functional information on myocardial perfusion as a "one-stop-shop" modality. In this context, recent studies have shown that the entire coronary tree can be extracted directly from the CTP dataset, preserving the imaging quality and the diagnostic accuracy in the detection of coronary plaques [20,21].

When speaking about dynamic CTP one of the main concerns remains the higher radiation dose compared to nuclear medicine modalities [22,23]. On average the mean effective dose reported for a single dynamic CTP scan was $9 \mathrm{mSv}$ [22]. Nevertheless, implementations of radiation dose reduction techniques, such as the use of low-tubevoltage settings [24] or the reduced number of scan acquisition for TACs construction [21], were able to reduce the radiation dose up to $40 \%$.

The manuscript by Bober et al. has further emphasized the need for a functional assessment of patients with stable coronary artery disease before coronary revascularization. In this context, despite the fact that dynamic CTP is still in its infancy and efforts have to be made to standardize scan acquisition and image analysis, it has the potential to enter 
into the clinical arena next to the well established functional imaging modalities.

Compliance with ethical standards Dr. A. Rossi and Dr. G. Ferrante declare they have no conflicts of interest.

\section{References}

1. Neumann F-J, Sousa-Uva M, Ahlsson A, Alfonso F, Banning AP, Benedetto U, et al. 2018 ESC/EACTS Guidelines on myocardial revascularization. Eur Heart J. 2019;40:87-165.

2. De Bruyne B, Pijls NHJ, Kalesan B, Barbato E, Tonino PAL, Piroth $Z$, et al. Fractional flow reserve-guided PCI versus medical therapy in stable coronary disease. N Engl J Med. 2012;367:991-1001.

3. Davies JE, Sen S, Dehbi H-M, Al-Lamee R, Petraco R, Nijjer SS, et al. Use of the instantaneous wave-free ratio or fractional flow reserve in PCI. N Engl J Med. 2017;376:1824-34.

4. Gotberg M, Christiansen EH, Gudmundsdottir IJ, Sandhall L, Danielewicz M, Jakobsen L, et al. Instantaneous wave-free ratio versus fractional flow reserve to guide PCI. N Engl J Med. 2017;376:1813-23.

5. De Bruyne B, Baudhuin T, Melin JA, Pijls NH, Sys SU, Bol A, et al. Coronary flow reserve calculated from pressure measurements in humans. Validation with positron emission tomography. Circulation. 1994;89:1013-22.

6. Johnson NP, Gould KL. Integrating noninvasive absolute flow, coronary flow reserve, and ischemic thresholds into a comprehensive map of physiological severity. JACC Cardiovasc Imaging. 2012;5: 430-40.

7. Johnson NP, Gould KL. Physiological basis for angina and STsegment change PET-verified thresholds of quantitative stress myocardial perfusion and coronary flow reserve. JACC Cardiovasc Imaging. 2011;4:990-8.

8. Bober RM, Milani RV, Oktay AA, Javed F, Polin NM, Morin DP. The impact of revascularization on myocardial blood flow as assessed by positron emission tomography. Eur J Nucl Med Mol Imaging. 2019;46(6):1226-1239.

9. Bober RM, Thompson CD, Morin DP. The effect of coronary revascularization on regional myocardial blood flow as assessed by stress positron emission tomography. J Nucl Cardiol. 2017;24:96174.

10. van de Hoef TP, Echavarria-Pinto M, van Lavieren MA, Meuwissen M, Serruys PWJC, Tijssen JGP, et al. Diagnostic and prognostic implications of coronary flow capacity: a comprehensive cross-modality physiological concept in ischemic heart disease. JACC Cardiovasc Interv. 2015;8:1670-80.

11. Bamberg F, Hinkel R, Schwarz F, Sandner TA, Baloch E, Marcus $\mathrm{R}$, et al. Accuracy of dynamic computed tomography adenosine stress myocardial perfusion imaging in estimating myocardial blood flow at various degrees of coronary artery stenosis using a porcine animal model. Investig Radiol. 2012;47:71-7.

12. Rossi A, Uitterdijk A, Dijkshoorn M, Klotz E, Dharampal A, van Straten M, et al. Quantification of myocardial blood flow by adenosine-stress CT perfusion imaging in pigs during various degrees of stenosis correlates well with coronary artery blood flow and fractional flow reserve. Eur Heart J Cardiovasc Imaging. 2013;14:331-8.

13. Pelgrim GJ, Duguay TM, Stijnen JMA, Varga-Szemes A, Van Tuij1 $\mathrm{S}$, Schoepf UJ, et al. Analysis of myocardial perfusion parameters in an ex-vivo porcine heart model using third generation dualsource CT. J Cardiovasc Comput Tomogr. 2017;11:141-7.

14. van Assen M, Pelgrim GJ, Slager E, van Tuijl S, Schoepf UJ, Vliegenthart R, et al. Low CT temporal sampling rates result in a substantial underestimation of myocardial blood flow measurements. Int J Cardiovasc Imaging. 2019;35:539-47.

15. Ishida M, Kitagawa K, Ichihara T, Natsume T, Nakayama R, Nagasawa N, et al. Underestimation of myocardial blood flow by dynamic perfusion CT: explanations by two-compartment model analysis and limited temporal sampling of dynamic CT. J Cardiovasc Comput Tomogr. 2016;10:207-14.

16. Rossi A, Wragg A, Klotz E, Pirro F, Moon JC, Nieman K, et al. Dynamic computed tomography myocardial perfusion imaging: comparison of clinical analysis methods for the detection of vessel-specific ischemia. Circ Cardiovasc Imaging. 2017;10(4).

17. Ho K-T, Ong H-Y, Tan G, Yong Q-W. Dynamic CT myocardial perfusion measurements of resting and hyperaemic blood flow in low-risk subjects with 128-slice dual-source CT. Eur Heart J Cardiovasc Imaging. 2015;16:300-6.

18. Ho K-T, Chua K-C, Klotz E, Panknin C. Stress and rest dynamic myocardial perfusion imaging by evaluation of complete timeattenuation curves with dual-source CT. JACC Cardiovasc Imaging. 2010;3:811-20.

19. Celeng C, Leiner T, Maurovich-Horvat P, Merkely B, de Jong P, Dankbaar JW, et al. Anatomical and functional computed tomography for diagnosing hemodynamically significant coronary artery disease: a meta-analysis. JACC Cardiovasc Imaging. 2018;S1936878X(18)30681-8.

20. Yi Y, Wu W, Lin L, Zhang H-Z, Qian H, Shen Z-J, et al. Singlephase coronary artery CT angiography extracted from stress dynamic myocardial CT perfusion on third-generation dual-source CT: validation by coronary angiography. Int J Cardiol. 2018;269:343-9.

21. Hubbard L, Lipinski J, Ziemer B, Malkasian S, Sadeghi B, Javan H, et al. Comprehensive assessment of coronary artery disease by using first-pass analysis dynamic CT perfusion: validation in a swine model. Radiology. 2018;286:93-102.

22. Danad I, Szymonifka J, Schulman-Marcus J, Min JK. Static and dynamic assessment of myocardial perfusion by computed tomography. Eur Heart J Cardiovasc Imaging. 2016;17:836-44.

23. Gimelli A, Achenbach S, Buechel RR, Edvardsen T, Francone M, Gaemperli O, et al. Strategies for radiation dose reduction in nuclear cardiology and cardiac computed tomography imaging: a report from the European Association of Cardiovascular Imaging (EACVI), the cardiovascular committee of European Association of Nuclear Medicine (EANM), and the European Society of Cardiovascular Radiology (ESCR). Eur Heart J. 2018;39:286-96.

24. Fujita M, Kitagawa K, Ito T, Shiraishi Y, Kurobe Y, Nagata M, et al. Dose reduction in dynamic $\mathrm{CT}$ stress myocardial perfusion imaging: comparison of $80-\mathrm{kV} / 370-\mathrm{mAs}$ and $100-\mathrm{kV} / 300-\mathrm{mAs}$ protocols. Eur Radiol. 2014;24:748-55.

Publisher's note Springer Nature remains neutral with regard to jurisdictional claims in published maps and institutional affiliations. 